\title{
Coil embolization of bronchial artery aneurysm with high flow bronchial artery-pulmonary artery fistula
}

\author{
Yoshiro Matsuo*, Takuya Okada, Masato Yamaguchi, Akhamadu Muradi, Teruaki Okuno, \\ Noriaki Sakamoto, Koji Idoguchi, Koji Sugimoto \\ Department of Radiology and Center for Endovascular Therapy, Kobe University Graduate School of Medicine, Kobe, Japan \\ Email: ${ }^{*}$ taillesspine@hotmail.com
}

Received 20 September 2012; revised 26 October 2012; accepted 16 November 2012

\begin{abstract}
We report a case of an asymptomatic 36-year-old man with a bronchial artery aneurysm in the right hilum. Selective angiography revealed a $25 \mathrm{~mm}$ saccular aneurysm and an efferent artery of the aneurysm forming a high flow bronchial artery-pulmonary artery fistula. Because of dilatation and tortuosity of the bronchial artery, the microcatheter could reach the efferent artery but not the fistula. Therefore, we embolized the fistula by sending microcoils through the bloodstream from the efferent artery to the fistula (the "flow-dependent" coil embolization technique), and further embolized the aneurysm by coil isolation and packing technique.
\end{abstract}

Keywords: Bronchial Artery Aneurysm; Bronchial Artery-Pulmonary Artery Fistula; Embolization

\section{INTRODUCTION}

Bronchial artery aneurysm is a rare condition, mainly caused by chronic inflammation or trauma [1]. It is usually diagnosed through symptoms such as hemoptysis, and its rupture can be life-threatening [2]. Recently, the number of reports on indications for transcatheter bronchial artery embolization is increasing [3]. However, the bronchial artery in this condition often appears enlarged and remarkably tortuous, which makes the catheterization difficult. The aneurysm is often accompanied with the bronchial artery-pulmonary artery fistula, which can relate to aneurysm formation. The embolization of the fistula can be further difficult because it is located distal to the aneurysm.

We report a case of successful embolization with coils for asymptomatic bronchial artery aneurysm with a highflow bronchial artery-pulmonary artery fistula.

*Corresponding author.

\section{CASE REPORT}

A 36-year-old man was transported to our hospital after a traffic accident. The patient was conscious with stable vital signs. He only complained of left clavicular pain due to the dislocation of left clavicle. Medical history was unremarkable and he was a 20-year smoker. Contrast-enhanced computed tomography (CT) revealed no traumatic change such as a hematoma in chest or abdomen, but the right bronchial artery was enlarged and remarkably tortuous (Figure 1(a)). Further, a $25 \mathrm{~mm}$ diameter aneurysm was noted in the right hilum, contiguous with the right bronchial artery (Figure 1(b)). Calcifications along the aneurysmal wall indicated that the aneurysm was not a false one due to the trauma, but a true aneurysm. Except mild hypertrophy of the bronchus, no abnormalities were detected in the lung region. The patient did not present with subjective symptoms such as hemorrhage; still, a possible aneurysm rupture would be life-threatening. The informed consent was obtained and transcatheter embolization of the bronchial artery aneurysm was carried out six days after initial diagnosis.

Angiography of the right bronchial artery showed a 25 $\mathrm{mm}$ saccular aneurysm. CT-angiography of the right bronchial artery showed that the efferent artery of the aneurysm formed a fistula with the right lower pulmonary artery (Figures 2(a) and (b)). The left bronchial artery was not apparently enlarged, but minute arteries proliferation and densely stained lung parenchyma were noted in the peripheral region of the lung. Tortuosity of the right bronchial artery prevented advancing the operating catheter. Therefore, a 7F guiding catheter originally made for right coronary artery (Brite Tip AL1 SH; Johnson \& Johnson Cordis, New Brunswick, New Jersey), and a 5F catheter (CX catheter A II ; CATHEX, Kanagawa, Japan) were advanced into the right bronchial artery to assist the operating catheter. Following, we catheterized the right bronchial artery with a $2.3 \mathrm{~F}$ microcatheter with two radiopaque tip markers (Carry; UTM Co., Aichi, Japan). 


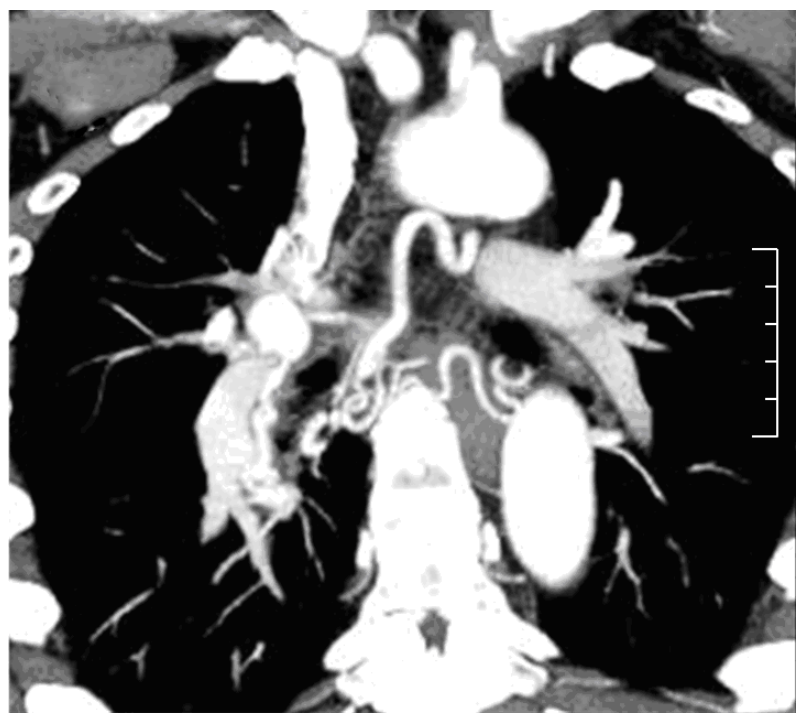

(a)

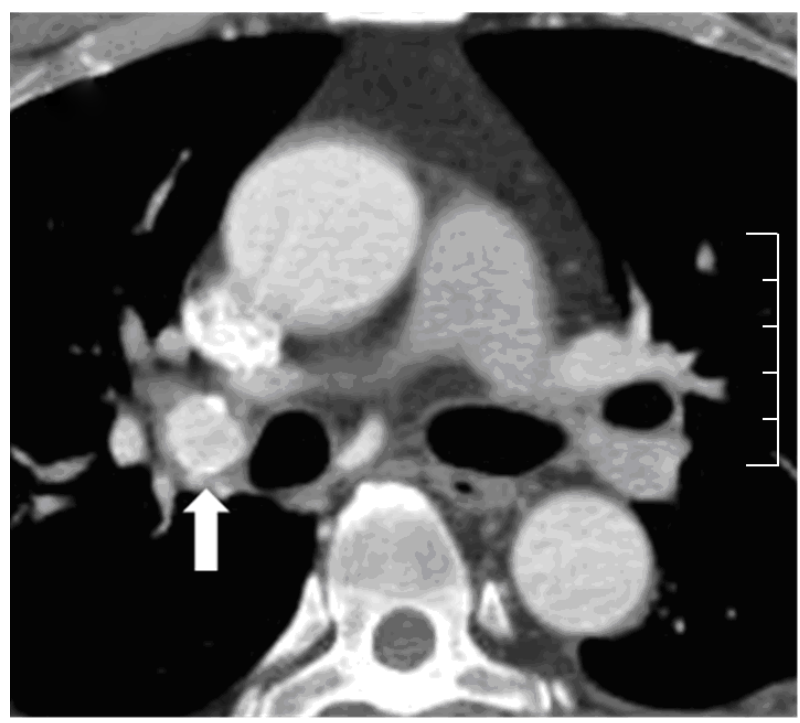

(b)

Figure 1. Initial contrast-enhanced CT. (a) Maximum Intensity Projection image shows remarkably enlarged and tortuous right bronchial artery; (b) Bronchial artery aneurysm (arrow) in the right hilum.

We achieved advancing catheter tip into the efferent artery, just beyond the aneurysm, but could not advance to the fistula with the right lower pulmonary artery. Although the fistula had high blood flow, the size was small. Therefore, we selected three 0.018-inch $3 \mathrm{~mm}$ microcoils (Tornade; Cook Inc., Bloomington, IN, USA) which were slightly smaller than the efferent artery (4 $\mathrm{mm}$ in diameter), but sufficiently larger than the fistula (1.2 $\mathrm{mm}$ in diameter). We performed embolization by sending the microcoils through the bloodstream (the "flow-dependent" coil embolization technique) (Figures 3(a) and (b)), and embolized the efferent artery with 0.018 inch $4 \mathrm{~mm}$ and $5 \mathrm{~mm}$ microcoils. Further, in the aneurysm

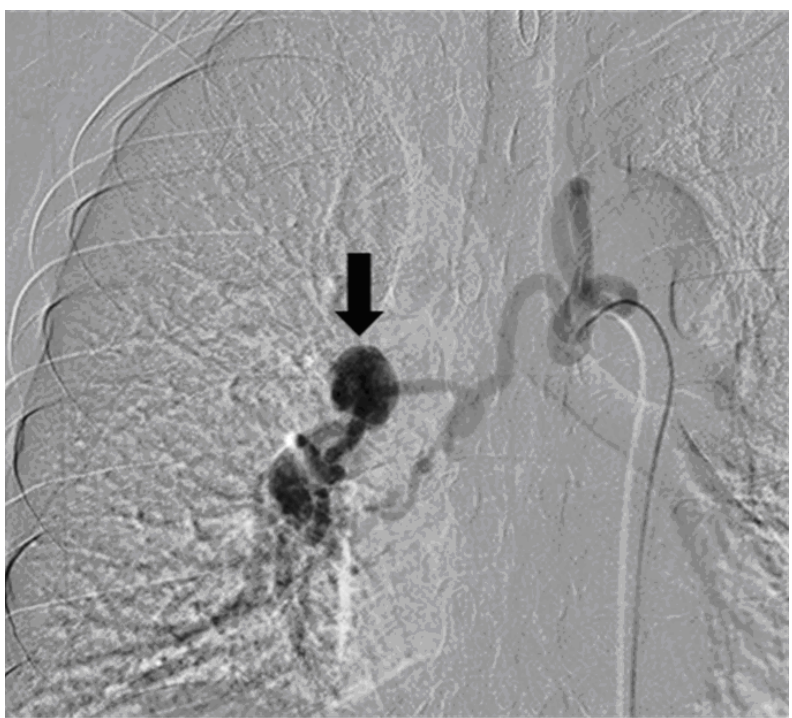

(a)

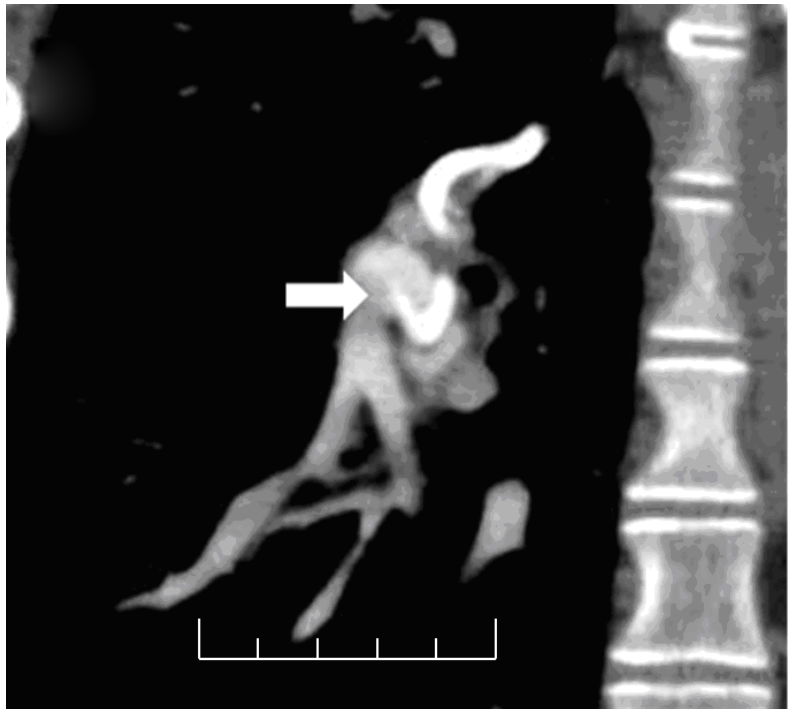

(b)

Figure 2. Angiography and CT angiography. (a) Angiography of the right bronchial artery shows remarkably enlarged and tortuous right bronchial artery, and a saccular aneurysm (arrow); (b) CT-angiography of the right bronchial artery shows an efferent artery of the aneurysm forming a fistula with the right lower pulmonary artery (arrow).

we established a framework with interlocking detachable coils (IDCs; Boston Scientific Japan Co., Tokyo), and packed 0.018 inch microcoils. We embolized the afferent artery, achieving the isolation. Post-procedural angiography showed the efferent artery appearing from collateral arteries originated from the right bronchial artery branch proximal to the aneurysm, but the aneurysm and the fistula were occluded. (Figure 3(c)). The patient was discharged with no complications, and the follow up was continued at the outpatient service. 21 months after transcatheter artery embolization, the patient works normally 


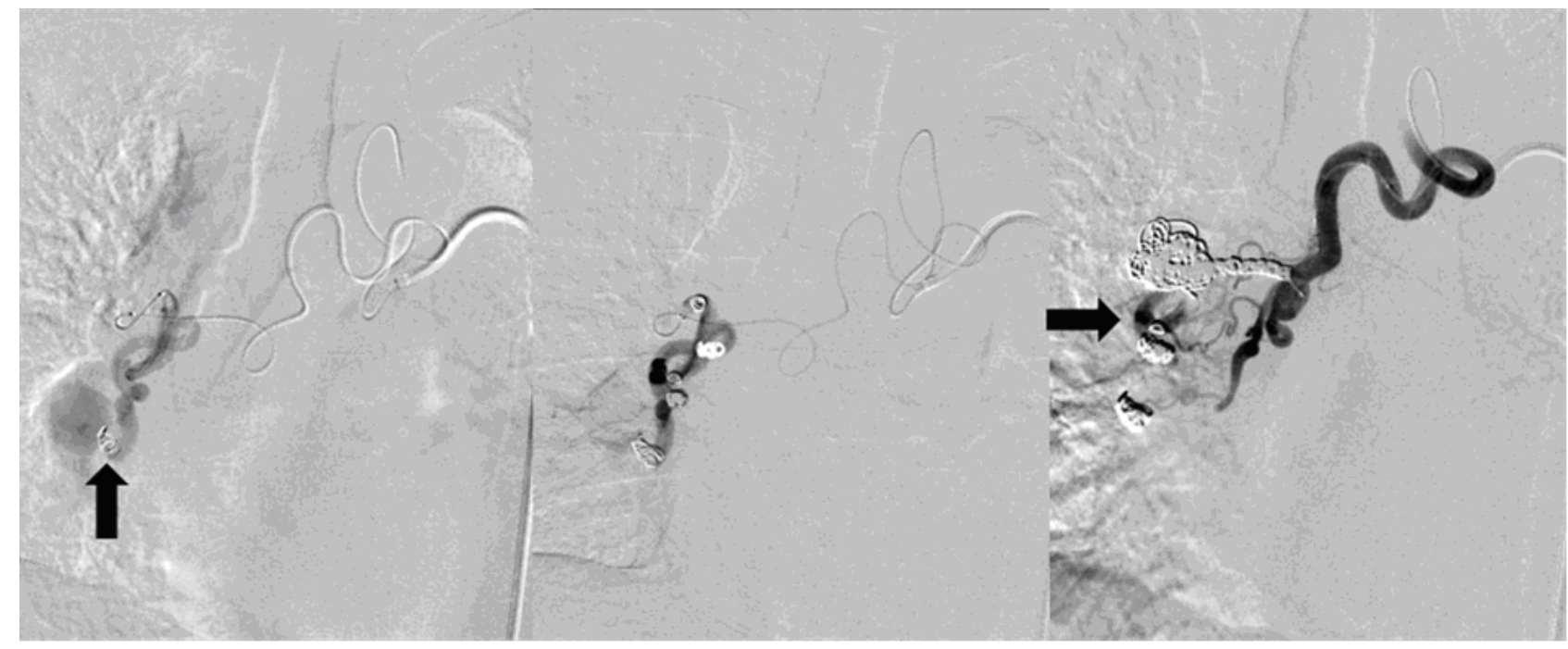

(a)

(b)

(c)

Figure 3. Embolization of bronchial artery aneurysm and bronchial artery-pulmonary artery fistula. (a) The "flow-dependent” coil embolization technique. The embolization of the fistula was performed by sending the microcoils through the bloodstream (arrow); (b) The pulmonary artery not visible and the fistula successfully embolized; (c) Post-procedural angiography shows the efferent artery appearing from collateral arteries originating from the right bronchial artery branch proximal to the aneurysm; the aneurysm and the fistula occluded (arrow).

without any symptoms.

\section{DISCUSSION}

It was reported that bronchial artery aneurysm was noted in approximately $0.7 \%$ of all patients who underwent selective bronchial arteriography for some condition, such as hemoptysis [4]. Little is known about the cause of bronchial artery aneurysm. According to Hoffman et al., underlying chronic lung diseases result in increasing bronchial artery flow, which leads to dilatation, tortuosity, and proliferation of bronchial artery, and subsequent aneurysm formation in weakening part of arterial wall [5]. Underlying diseases include pulmonary sequestration and pulmonary agenesis [6] as congenital disease, and atherosclerosis, bronchiectasis, chronic inflammation, infection, and trauma [1] as acquired disease. Some reports describe cases associated with systemic vascular abnormalities, such as Rendu-Osler-Weber syndrome [7] and sepsis [8]. In our case, there were no underlying disease and medical histories such as pneumonia in childhood. However, our patient was a heavy smoker, and CT showed mild hypertrophy of the bronchus, while angiography from a bronchial artery showed proliferated vessels and densely stained lung parenchyma. Thus, we suspected that some chronic respiratory inflammation caused forming of the bronchial artery-pulmonary artery fistula, which led to increasing bronchial artery flow and resulted in aneurysm formation.

Rupture of aneurysm can be life-threatening, and previous reports indicate that bronchial artery aneurismal diameter may not be an incremental risk factor. Therefore, even when no symptoms were present, most cases were treated to prevent rupture. Traditional treatment approaches mainly consisted of surgical extirpation. Recently, transcatheter bronchial artery embolization is becoming widely accepted as it is less invasive, and should be the first choice treatment.

In our case, the high flow bronchial artery-pulmonary artery fistula was accompanied by the aneurysm, indicating that the fistula resulted in increasing bronchial artery flow. We suspected that embolizing only the aneurysm leaves the risk of another aneurysm formation in the branches or possible collateral arteries of the bronchial artery, which were actually confirmed on the postprocedural angiography. Therefore, both the aneurysm and the fistula were embolized.

In bronchial artery aneurysm, the artery itself is often enlarged and remarkably tortuous, causing difficulties in catheterization. Previous reports described managing similar situation with N-butyl-2-cyanoacrylate (NBCA) $[9,10]$. In our case, catheter could reach the efferent artery but not to the fistula. As the fistula was small but with high-flow, the embolization with liquid and particle embolic materials involved the risk of severe complication secondary to migration to the pulmonary artery. Thus, "flow-dependent" coil embolization was performed. The "flow-dependent" embolization means that the embolic material will be carried by the bloodstream to occlude remotely from the catheter tip. This technique is conventionally performed with liquid or particle embolic materials. However, when embolizing a fistula, coils of 
appropriate size are optimal material.

\section{REFERENCES}

[1] Cearlock, J.R., Fontaine, A.B., Urbaneja, A., et al. (1995) Endovascular treatment of a posttraumatic bronchial artery pseudoaneurysm. Journal of Vascular and Interventional Radiology, 6, 495-496. doi:10.1016/S1051-0443(95)72852-4

[2] Osada, H., Kawada, T., Ashida, H., et al. (1986) Bronchial artery aneurysm. The Annals of Thoracic Surgery, 41, 440-442. doi:10.1016/S0003-4975(10)62706-3

[3] Kasashima, F., Endo, M., Kosugi, I., et al. (2003) Mediastinal bronchial artery aneurysm treated with a stentgraft. Journal of Endovascular Therapy, 10, 381-385. doi:10.1583/1545-1550(2003)010<0381:MBAATW >2.0. $\underline{\mathrm{CO} ; 2}$

[4] Tanaka, K., Ihaya, A., Horiuci, T., et al. (2003) Giant mediastinal bronchial artery aneurysm mimicking benign esophageal tumor: A case report and review of 26 cases from literature. Journal of Vascular Surgery, 38, 11251129. doi:10.1016/S0741-5214(03)00707-9

[5] Hoffman, V., Ysebaert, D., De Schepper, A., et al. (1996) Acute superior vena cava obstruction after rupture of a bronchial artery aneurysm. Chest, 110, 1356-1358. doi:10.1378/chest.110.5.1356

[6] Sancho, C., Dominguez, J., Escalante, E., et al. (1999) Embolization of an anomalous bronchial artery aneurysm in a patient with agnesis of the left pulmonary artery. Journal of Vascular and Interventional Radiology, 10, 1122-1126. doi:10.1016/S1051-0443(99)70202-2

[7] Kalangos, A., Khatchatourian, G., Panos, A., et al. (1997) Ruptured mediastinal bronchial artery aneurysm: A dilemma of diagnosis and therapeutic approach. The Journal of Thoracic and Cardiovascular Surgery, 114, 853856. doi:10.1016/S0022-5223(97)70094-1

[8] Chantepie, A., Robert, M., Pelletier, J., et al. (1980) Mycotic aneurysm of bronchial artery: Apropos of a case in an infant. Cirugía Pediátrica, 21, 407-410.

[9] Lu, P.H., Wang, L.F., Su, Y.S., et al. (2011) Endovascular therapy of bronchial artery aneurysm: five cases with six aneurysms. CardioVascular and Interventional Radiology, 34, 508-512. doi:10.1007/s00270-010-9895-X

[10] Aburano, H., Kawamori, Y., Horiti, Y., et al. (2006) Bronchial artery aneurysm embolization with NBCA. CardioVascular and Interventional Radiology, 29, 11411143. doi:10.1007/s00270-005-0166-1 